\title{
Cisplatin/Doxorubicin/Ifosfamide Regimen
}

National Cancer Institute

\section{Source}

National Cancer Institute. Cisplatin/Doxorubicin/Ifosfamide Regimen. NCI Thesaurus.

Code C9600.

A chemotherapy regimen consisting of cisplatin, doxorubicin, and ifosfamide that may be used in the treatment of certain osteo- and uterine sarcomas. 